\title{
CARACTERIZAÇÃO DE CONTAMINAÇÃO POR ENTEROPARASITAS EM AMOSTRAGEM DE ALFACES COMERCIALIZADAS NO INTERIOR PAULISTA
}

\author{
LOPES, Marcos Eduardo Ramos ${ }^{1}$ \\ SANTOS, Hertz Figueiredo dos ${ }^{2}$
}

RESUMO: O Consumo de hortaliças se torna uma das principais vias de transmissão de enteroparasitas (parasitas de intestino) devido as diferentes formas de contaminação que estão sujeitas. O presente trabalho teve como objetivo investigar a possível presença de enteroparasitas, protozoários e helmintos em alfaces (Lactuca sativa) comercializadas em hortas de três cidades do interior paulista: Ipuã, Ituverava e Miguelópolis. As 25 amostras de cada cidade foram devidamente transportadas e analisadas no laboratório de análises clínicas da FAFRAM. Das 75 amostras, $15(20 \%)$ foram positivos para enteroparasitas, destas, uma $(1,4 \%)$ foi observada a presença de cistos de protozoários, catorze $(18,6 \%)$ foram detectados ovos/larvas de helmintos. Desse modo, das três cidades, a que menos apresentou contaminação nas amostras por algum enteroparasita foi Miguelópolis, com apenas uma amostra contaminada por Entamoeba coli $(4 \%)$ das 25 analisadas. Já a cidade que apresentou maior contaminação foi Ipuã, com 11 amostras contaminadas, onde cinco amostras estavam contaminadas com Strongyloides stercoralis (20\%), três amostras contaminadas com Diphyllobothrium latum (12\%), duas amostras contaminadas com Ancilostomideos (8\%) e uma amostra contaminada com um helminto não identificado (4\%) das 25 analisadas. Já Ituverava apresentou duas amostras contaminadas com Strongyloides stercoralis (8\%) e uma amostra contaminada com Ancilostomídeos (4\%) das 25 amostras. Levando em conta os resultados obtidos, ressalta-se a importância das hortaliças consumidas cruas na transmissão dos enteroparasitas, bem como a necessidade de medidas que propiciem uma melhoria na qualidade higiênico-sanitária desses alimentos.

RESUMEN: El consumo de hortalizas se convierte en una de las principales vías de transmisión de enteroparásitos (parásitos intestinales) debido a las diferentes formas de contaminación a las que están sujetos. El presente trabajo tuvo como objetivo investigar lo posible presencia de enteroparásitos, protozoos y helmintos, en lechugas (Lactuca sativa) comercializadas en huertos de tres ciudades del interior paulista: Ipuã, Ituverava y Miguelópolis, Las 25 muestras de cada ciudad fueron llevadas al laboratorio de análisis clínico de la FAFRAM. De las 75 muestras, 15 (20\%) fueron positivas para enteroparásitos, de estas, en una $(1,4 \%)$ fue observada la presencia de quistes de protozoos y en catorce $(18.6 \%)$ fueron detectados huevos/larvas de helmintos. Por lo tanto, de las tres ciudades, la que presentó menos contaminación en las muestras por algún enteroparásito fue Miguelópolis, con solo una muestra contaminada por Entamoeba coli (4\%) de las 25 analizadas. La ciudad que presentó mayor contaminación fue Ipuã, con 11 muestras contaminadas, donde cinco muestras estaban contaminadas con Strongyloides stercoralis (20\%), tres muestras contaminadas con Diphyllobothrium latum (12\%), dos muestras contaminadas con Anquilostomas (8\%) y una. muestra contaminada con un helminto no identificado (4\%) de las 25 analizadas. Ya Ituverava presentó dos muestras contaminadas con Strongyloides stercoralis (8\%) y una muestra contaminada con Anquilostoma (4\%) de las 25 muestras. Teniendo en cuenta los resultados obtenidos, se enfatiza la importancia de las hortalizas consumidas crudas en la transmisión de enteroparásitos, así como la necesidad de medidas que proporcionen una mejora en la calidad higiénico-sanitaria de estos alimentos.

Palavras-chave: Protozoários. Helmintos. Verduras.

\section{INTRODUÇÃO}

A presença de uma elevada incidência de doenças parasitárias de grande importância de saúde pública, afetam principalmente, as comunidades mais pobres de países em desenvolvimento. Atingindo todas as faixas etárias, essas doenças causam os mais diversos sintomas, como anemia, diarreia, má absorção dos nutrientes, emagrecimento, diminuição da capacidade de aprendizado entre outros

\footnotetext{
${ }^{1}$ Biólogo FFCL/FE -Mestrando em Genética pela Faculdade de Medicina da USP de Ribeirão Preto - FMRP-USP ${ }^{2}$ Biólogo FFCL/USPRP. Doutor em Zoologia . Docente FFCL/FE- Ituverava; Faculdade São Luis - Jaboticabal.
} 
(FARIA et al., 2008, PAULA et al., 2003).

O consumo de hortaliças se torna uma das principais vias de transmissão de enteroparasitas (parasitas intestinais) devido a frequente prática de irrigação de hortas com água contaminada por material fecal (TAKAYANAGUI et al., 2001), expondo assim grande parte da população às formas de transmissão de diversas enfermidades intestinais.

De acordo com Falavigna et al (2005), uma forma comum de aquisição de parasitoses é pela ingestão de alimentos crus contaminados por cistos, ovos ou larvas, como os vegetais. O solo é contaminado por essas estruturas parasitárias, através de dejetos eliminados pelo homem e animais parasitados. Assim, a água pode preservar esses elementos parasitários por longos períodos e transportálos a grandes distâncias.

O desenvolvimento de estágios infectantes ocorre no solo, e quando os alimentos vegetais são consumidos crus, os trazem de volta ao hospedeiro suscetível, o homem. (MARZOCHI; CARVALHEIRO, 1978). Desta forma, o homem é um dos principais vetores ou reservatórios do processo de contaminação dos alimentos por parasitas (REZENDE et al., 1997). Algumas parasitoses que podem ser transmitidas por verduras cruas contaminadas são: Amebíase (Entamoeba coli e Entamoeba histolytica), Ascaridíase (Ascaris lumbricoides), Giardíase (Giardia lamblia), entre outras (NEVES et al., 2005).

As verduras mais consumidas cruas pela população são alfaces e agriões (NORBERG et al, 2008), entretanto, pelo fato de ser consumida crua, a alface serve de fonte para a transmissão de diferentes espécies de parasitos (RUL, SENER, 2005). Segundo Traviezo -Valles et al. (2004) a hortaliça de consumo cru com maior índice de contaminação enteroparasitária é a alface, tendo assim grande importância na saúde humana.

Dessa forma, os enteroparasitas são veiculados em sua maior parte por água e alimentos contaminados, contudo, é importante avaliar os hábitos higiênicos e realizar constantes inquéritos parasitológicos na população ${ }^{1}$ (SANTOS; CABRERA, 2011). Na epidemiologia da transmissão de enteroparasitoses, é levado em consideração os mais diversos fatores que estão relacionados com os meios de contaminação, como por exemplo mãos sujas, alimentos em geral, moscas, outros insetos, ar e água (VALLADA et al., 1987; BRANCO et al. 1999).

Uma boa higiene é medida fundamental contra doenças oriundas de alimentos, e o indivíduo que fornece, prepara e serve o alimento, também deve construir barreiras sanitárias entre este e seus consumidores. O desenvolvimento de postura crítica como consumidor desses alimentos também é fundamental para atingir a produção de alimentos seguros, e depende fundamentalmente de investimentos em educação (SOARES; CANTOS, 2006).

Para manutenção dos níveis aceitáveis para o consumo, as autoridades devem ter o compromisso de fiscalizar os produtores. A organização Mundial da Saúde - OMS, estabelece um limite de ovos de nematoides em águas de irrigação dos vegetais, sendo menor ou igual a um ovo/ litro (FREITAS, 2004).

Alguns trabalhos como o de Garcia et al (2004) analisaram 133 amostras de hortaliças no município de Umuarama, onde foram encontrados Enterobius vermicularis (1,5\%), Strongyloides sp (0,8\%), Entamoeba sp (3,8\%), Ascaris sp (9,7\%), Ancilostomídeos (9,7\%) e Giardia sp (0,8\%).

Diante do exposto, esse trabalho objetivou avaliar, do ponto de vista parasitológico, alfaces comercializadas em hortas de três cidades do interior paulista. 


\section{MATERIAL E MÉTODO}

Foram coletadas e analisadas amostras de alfaces (Lactuca sativa) comercializadas em três cidades do interior paulista: Ipuã, Ituverava e Miguelópolis, no período de abril a setembro de 2017. Sendo 25 de cada cidade, totalizando 75 amostras. Estabeleceu-se como unidade amostral para alface, 10 folhas independentemente do peso ou tamanho. As amostras escolhidas de formas aleatórias eram acondicionadas em sacolas plásticas próprias das hortas onde foram compradas. Para evitar contaminações adicionais, as sacolas foram lacradas e só foram abertas no laboratório.

As análises foram realizadas no laboratório de Análises Clínicas do Hospital Veterinário da FAFRAM - Faculdade Doutor Francisco Maeda, no município de Ituverava-SP. No laboratório, foram separadas do pé, as folhas, desprezando-se aquelas deterioradas, bem como o talo, (OLIVEIRA et al., 2012). Em uma bandeja, as hortaliças foram mergulhadas em solução de detergente neutro, contendo duas gotas de detergente diluído em $250 \mathrm{ml}$ de água destilada. Com o auxílio de um pincel chato $\left(\mathrm{n}^{\mathrm{o}} 16\right)$, esfregava-se toda a superfície das folhas, deixando-as por 30 segundos em repouso, e depois, as folhas eram levantadas para escorrer o excesso de líquido, e em seguida, eram desprezadas. Esta operação foi repetida, com as demais folhas, até que toda a amostra fosse completamente lavada (OLIVEIRA; GERMANO, 1992).

O líquido obtido foi filtrado em gaze de quatro dobras sobre uma peneira e recolhido em um frasco cônico onde foi deixado por 24 horas para sedimentação, de acordo com a metodologia de Hoffman, Pons e Janer (1934) adaptado. Completada a sedimentação, foi recolhido cerca de $1 \mathrm{ml}$ do sedimento com uma pipeta de Pasteur, e colocada na lâmina para microscopia, corado com lugol, e analisado no microscópio óptico em duplicata. As formas infectantes dos parasitos foram identificadas de acordo com Cimerman e Franco, (2002) Neves e Bittencourt-Neto (2008).

\section{RESULTADO E DISCUSSÃO}

Na Tabela 1, apresenta-se a ocorrência dos protozoários e dos helmintos nas alfaces analisadas. Das 75 amostras, $15(20,00 \%)$ foram positivos para enteroparasitas, destes, uma $(6,7 \%)$ foi observada a presença de cistos de protozoários, e catorze $(93,3 \%)$ foram detectados ovos/larvas de helmintos

Tabela 1: Prevalência de positividade para protozoários e helmintos em 75 amostras de alfaces analisadas e comercializadas em três municípios do interior de São Paulo: Ipuã. Ituverava e Miguelópolis.

\begin{tabular}{lcc}
\hline Positivas para: & $\begin{array}{c}\text { Presença de } \\
\text { Enteroparasitas }\end{array}$ & $\begin{array}{c}\text { Total de amostras } \\
\text { analisadas }\end{array}$ \\
\hline Protozoários & 01 & $1,4 \%$ \\
\hline Helmintos & 14 & $18,6 \%$ \\
\hline Total & 15 & $20 \%$ \\
\hline
\end{tabular}

Fonte: Elaborada pelo Autor.

A frequência de estruturas parasitárias (15/75 - 20,00\%) encontrada no presente estudo caracteriza o papel da alface como via de transmissão para os seres humanos. Amostras contaminadas com ovos de Ancilostomídeos indicam a contaminação com material fecal oriundo do homem, cães e até gatos (COSTA, 2012). 
$\mathrm{Na}$ Tabela 2 verifica-se a prevalência de enteroparasitas por cidade. Em Miguelópolis, das 25 amostras analisadas, apenas uma (4\%) apresentou contaminação. Já em Ituverava, das 25 amostras analisadas, três (12\%) apresentaram contaminação, e em Ipuã, das 25 amostras analisadas, 11 amostras (44\%) apresentaram contaminação.

Dessas três cidades, a que menos apresentou contaminação por algum enteroparasita foi Miguelópolis, com apenas uma amostra contaminada com Entamoeba coli (4\%) das 25 analisadas. Já a cidade que apresentou maior contaminação foi Ipuã, com 11 amostras contaminadas, onde cinco amostras estavam contaminadas com Strongyloides stercoralis (20\%), três amostras contaminadas com Diphyllobothrium latum (12\%) e duas amostras contaminadas com Ancilostomideos (8\%), e uma amostra contaminada com um helminto não identificado (4\%), das 25 analisadas. A cidade Ituverava apresentou duas amostras contaminadas com Strongyloides stercoralis (8\%) e uma amostra contaminada com Ancilostomideos (4\%) das 25 analisadas.

Tabela 2: Prevalência de helmintos e protozoários por cidade avaliada.

\begin{tabular}{lccc} 
& \multicolumn{3}{c}{ Número e porcentagem de amostras contaminadas por cidade } \\
\cline { 2 - 4 } & Ipuã & Ituverava & Miguelópolis \\
\hline Parasitas & & & \\
\hline Ancilostomídeos & $02(8 \%)$ & $01(4 \%)$ & $0(0 \%)$ \\
\hline Diphyllobothrium latum & $03(12 \%)$ & $0(0 \%)$ & $0(0 \%)$ \\
\hline Entamoeba coli & $0(0 \%)$ & $0(0 \%)$ & $1(4 \%)$ \\
\hline Strongyloides stercolaris & $05(20 \%)$ & $02(8 \%)$ & $0(0 \%)$ \\
\hline Helminto não identificado & $01(04 \%)$ & $0(0 \%)$ & $0(0 \%)$ \\
\hline \multicolumn{1}{c}{ Total } & $11(44 \%)$ & $03(12 \%)$ & $02(8 \%)$ \\
\hline \multicolumn{1}{c}{} & Fonte: Elaborada pelo Autor.
\end{tabular}

De acordo com Leite (2005 apud NEVES et al.,2005), dentre as mais de 100 espécies da família Ancylostomidae descritas, apenas três são agentes etiológicos das ancilostomoses humanas: Ancylostoma duodenale, Necator americanus e Ancylostoma ceylanicum. Os resultados obtidos nesse trabalho corroboram com resultados obtidos no trabalho de Vollkopf, Lopes, Navarro (2006) onde também encontraram Strongyloides sp e Ancilostomatidae em amostras de alfaces comercializadas em Porto Murtinho - MS. Porém, resultados obtidos no trabalho de Soares e Canto (2006), diferiram do presente trabalho, pois foram encontradas estruturas parasitárias de Giardia spp, Endolimax nana, Ascaris lumbricoides, Trichiuris trichiura entre outros parasitas, em alfaces comercializadas na cidade de Florianópolis - SC.

Os resultados obtidos em um trabalho de avaliação parasitológica de alfaces em Fronteira Oeste RS também se mostraram positivos para Entamoeba sp, corroborando com os achados do nosso trabalho (SILVA et al., 2015). A presença de amebas como Entamoeba coli e ou Endolimax nana, apesar de não serem patogênicas, servem de indicador de contaminação de hortaliça por fezes humanas (OLIVEIRA; GERMANO, 1992).

A grande maioria das amostras infectadas estavam contaminadas com uma espécie de parasita por amostra, mas tiveram amostras que estavam contaminadas por até duas espécies de parasitas, como por exemplo ovos de Ancilostomídeo e larva de Strongyloides stercolaris encontrados na mesma amostra. No trabalho de Gregório et al (2012), fazendo um estudo da contaminação por enteroparasitas em hortaliças da região leste de São Paulo, acabaram encontrando também Diphillobothrium latum. 
É válido ressaltar que a contaminação pode ocorrer durante o processo de cultivo da hortaliça, quando há a utilização de adubo orgânico, água e solo contaminados, e também, no momento de armazenagem, transporte e manipulação/preparo feito por vendedores (TAKAYANAGUI et al., 2001), compradores, e também por pessoas que manipulam/preparam o alimento.

Trabalhos relatam a ocorrência de elementos parasitários também em outras hortaliças como no Agrião (Nasturtium officinale) (MOREIRA et al., 2017), Rúcula (Eruca vesicaria sativa) (FERNANDES et al., 2014) e Couve (MOURA et al., 2016), mostrando assim a importância de diferentes hortaliças na transmissão dessas enteroparasitoses. Dessa maneira, é essencial que a fiscalização sanitária atue de maneira mais rígida, e que as pessoas passem por um processo educativo de conscientização, no qual orientações quanto às corretas condições higiênico-sanitárias sejam fornecidas, constituindo um trabalho preventivo.

A Tabela 3 apresenta uma comparação de parasitos por agente etiológico. O agente etiológico com maior frequência de contaminação foi Strongyloides stercoralis com 9,33\% de frequência, e o agente com menor frequência foi a Entamoeba coli e o helminto não identificado com 1,33\% de frequência cada. Já Diphyllobothrium latum e Ancilostomideos ficaram igualmente com frequência de $4 \%$ cada.

Tabela 3: Prevalência de enteroparasitas por agente etiológico obtido.

\begin{tabular}{ccc}
\cline { 2 - 3 } & Amostras contaminadas & Porcentagem \\
\hline Ancilostomideos & 03 & $4 \%$ \\
\hline Diphyllobothrium latum & 03 & $4 \%$ \\
\hline Entamoeba coli & 01 & $1,33 \%$ \\
\hline Strongyloides stercoralis & 07 & $9,33 \%$ \\
\hline Helminto não identificado & 01 & $1,33 \%$ \\
\hline
\end{tabular}

Fonte: Elaborada pelo Autor.

Fotografia 1 - A: Ovo de Ancilostomídeo. B: Ovo de Diphyllobotrium latum. C: Larva Rabditóide de Strongyloides stercoralis.

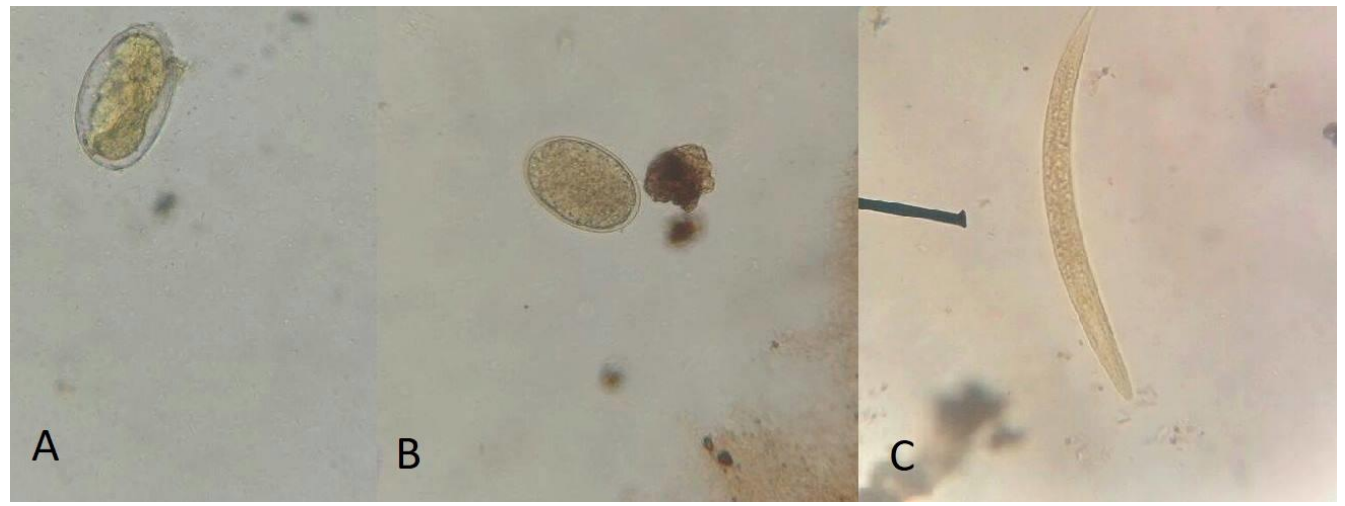

Fonte: Arquivo pessoal, 2017. 
Fotografia 2 - A e B: Helminto não identificado.

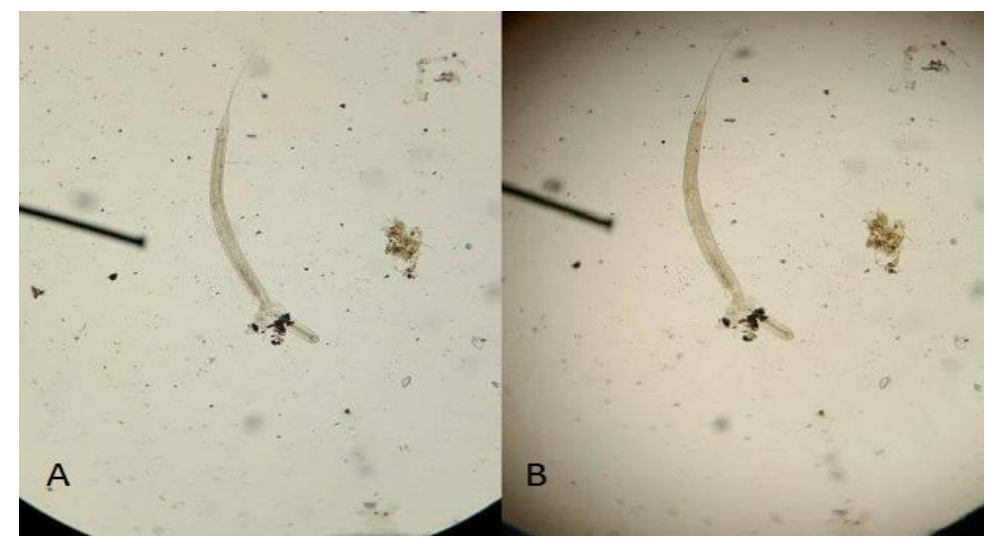

Fonte: Arquivo pessoal, 2017. Imagem A e B: Helminto não identificado. Imagem C: Ácaro

\section{CONCLUSÃO}

Das 75 amostras, 15 (20\%) foram positivos para enteroparasitas, destas, uma $(1,4 \%)$ foi observada a presença de cistos de protozoários, catorze $(18,6 \%)$ foram detectados ovos/larvas de helmintos. Das três cidades, a que menos apresentou contaminação nas amostras por algum enteroparasita foi Miguelópolis, com apenas uma amostra contaminada por Entamoeba coli (4\%) das 25 analisadas, e a cidade que apresentou maior contaminação foi Ipuã, com 11 amostras contaminadas, onde cinco amostras estavam contaminadas com Strongyloides stercoralis (20\%), três amostras contaminadas com Diphyllobothrium latum (12\%), duas amostras contaminadas com Ancilostomideos (8\%) e uma amostra contaminada com um helminto não identificado (4\%) das 25 analisadas. Já Ituverava apresentou duas amostras contaminadas com Strongyloides stercoralis (8\%) e uma amostra contaminada com Ancilostomídeos (4\%) das 25 amostras. Dessa forma, os achados sugerem que, em algum momento na produção dessas hortaliças, houve a contaminação por fezes de seres humanos e/ou de animais.

É sempre válido ressaltar a importância de uma higienização adequada, não só desses alimentos que são consumidos in natura, mas também de todos os outros. Além disso, o processo educativo de conscientização da população em geral e a atuação dos órgãos competentes de fiscalização da produção desses alimentos exercem papéis-chave no combate às enteroparasitoses.

\section{REFERÊNCIAS}

BRANCO-JR, A. C.; WAIB, C.M; FILHO, O. C. O. Importância da higiene dos Alimentos na epidemiologia das helmintoses. Ocorrência de ovos de helmintos em hortaliças. Revista Brasileira de Análises Clínicas, v.31, n 1, p. 124-126, 1999.

CIMERMAN, B.; FRANCO, M. A. Atlas de Parasitologia: artrópodes, protozoários e helmintos. São Paulo: Atheneu, 2000. p 2002.

COSTA, J. R. B. Ocorrência de enteroparasitos em amostras de alface (Lactuca sativa L.) comercializadas na feira livre de Pocinhos - PB. 2012. 35f. Trabalho de Conclusão de Curso (Graduação em Ciências Biológicas). Universidade Estadual da Paraíba, Campina Grande, 2012. 
FALAVIGNA, L.M. et al. Qualidade de hortaliças comercializadas no noroeste do Paraná, Brasil. Parasitologia Latinoamericana, v. 60, n. 3-4, p. 144-149, 2005. ISSN 0707-7712

FARIA, G. F. et al. Frequiência de enteroparasitos em amostras de alfaces (Lactuca sativa) comercializadas em feiras livres na cidade de Ipatinga, Minas Gerais. Rev Digital de Nutrição [Internet]. v.36, n.5, p.621-623, 2003. Disponível em: http://www.scielo.br/pdf/rsbmt/v36n5/a14v36n5.pdf

FERNANDES, K. C. et al. Contaminação por enteroparasitos em verduras de supermercados e feiras de Umuarama-PR. Arq. Ciênc. Vet. Zool. UNIPAR, Umuarama, v. 17, n. 2, p. 115-119, abr./jun. 2014.

FREITAS, A. A. et al. Avaliação parasitológica de alfaces (Lactuca sativa) comercializadas em feiras livres e supermercados do município de Campo Mourão, Estado do Paraná. Acta Scientiarum. Biological Sciences. Maringá, v. 26, n. 4, p. 381- 384, 2004

GARCIA, J, L; JAHN, T. R.; FERMO, E. E.; NEVES, U. S. das; PURETZ, I. Helminthes and protozoa occurrence in raw vegetables produced in Umuarama, Parana State. Arquivos de Ciencias Veterinarias e Zoologia da Unipar, Umuarama, v. 7, n. 1, p. 7-10, jan./jun. 2004.

HOFFMANN, W. A.; PONS, J. A.; JANER, J. L. Sedimentation concentration method in schistosome. Puerto Rico Journal of Public Health and Tropical Medicine, 1934.

LEITE, A. C. R, Ancylostomidae. In: NEVES, D. P.; MELO, A. L.; GENARO, O.; LINARDI, P, M. Parasitologia Humana. 11. ed. Rio de Janeiro: Atheneu, 2005. p.261 - 269.

MARZOCHI, M. C. CARVALHEIRO, J. R. 1978. Estudos dos fatores envolvidos na disseminação dos enteroparasitas. Rev Inst Med Trop São Paulo 20: 31-35.

MOREIRA, C. C. et al. Avaliação microbiológica e parasitológica de hortaliças comercializadas na baixada fluminense, Rio de Janeiro. Revista Uniabeu. v. 10, n. 26, set-dez. 2017

MOURA, R. L. SANTOS, T. VIEGAS, A. A. Avaliação parasitológica em Lactuca sativa (Alface) e Brassica oleracea 1. (Couve) procedentes da ceasa no município de Anápolis - GO. Revista educação em saúde, v. 4. n. 1. 2016

NEVES, D.P.; BITTENCOURT NETO, J.B. Atlas didático de parasitologia. 2ed. Rio de Janeiro: Atheneu, 2008.

NEVES, D. P. et al. Parasitologia Humana. 11 ed. São Paulo: Atheneu, 2005.

NORBERG, A. N.; et al. Prevalência de ovos, larvas, cistos e oocistos de elementos parasitários em hortaliças comercializadas no município de Nova Iguaçu, Rio de Janeiro, Brasil. Revista de Ciência \& Tecnologia, Nova Iguaçu, v. 8, n.1, p. 12-21, jun. 2008.

OLIVEIRA, C. A. F.; GERMANO, P. M. L. Estudo da ocorrência de enteroparasitas em hortaliças comercializadas na região metropolitana de São Paulo, Brasil. I - Pesquisa de helmintos. Revista Saúde Pública, v. 26, n. 4, p. 383-89, 1992.

OLIVEIRA, D. S. C.; BRITO, J. K; MAIA, M. C. Avaliação parasitológica em amostras de alface (Lactuca Sativa) comercializadas em supermercados de Ipatinga, Minas Gerais. Nutrir Gerais, Ipatinga, v. 6 n. 11, p. 933-944, ago./dez. 2012.

PAULA P, et al. Contaminação microbiológica e parasitológica em alfaces (Lactuca sativa) de restaurantes self-service, de Niterói, RJ. Rev Soc Bras Med Trop v.36, p.535-537, 2003.

REZENDE, C. H. A.; COSTA-CRUZ, J. M.; GENNARI-CARDOSO, M. L. Enteroparasitoses em manipuladores de alimentos de escolas públicas em Uberlândia (MG), Brasil. Rev. Panam. Salud Pública, v.2, n.6, p. 392-7, 1997. 
RUL, O. E.; SENER, H. The contamination of various fruit and vegetable with Enterobius vermicularis, Ascaris eggs, Entamoeba histolytica cysts and Giardia cysts. Food Control, v. 16, p. 557- 560, 2005

SANTOS, F. F.; CABRERA, G. P. B. A alface (Lactuca sativa) como fonte de infecção por enteroparasitas em alguns municípios brasileiros. Univali, 2011. Disponível em:

http://www.pergamum.univale.br/pergamum/tcc/Aalfacelactucasativacomofontedeinfeccaoporenteroparasi tasemalgunsmunicipiosbrasileiros.pdf

SANTOS, F.; CABRERA, G. P. A alface (Lactuca sativa) como fonte de infecção por enteroparasitas em alguns municípios brasileiros. 2011. PhD Thesis. Tesis de Maestría. Minas Gerais: Universidade Vale do Rio Doce.

SILVA, M. R. P. et al. Avaliação parasitológica de alfaces comercializadas em um município da fronteira oeste, Rio Grande do Sul, Brasil. Rev Patol Trop v. 44, n. 2, p. 163-169. abr.-jun. 2015.

SOARES, B.; CANTOS, G.A. Detecção de estruturas parasitárias em hortaliças comercializadas na cidade de Florianópolis, SC, Brasil. Revista Brasileira de Ciências Farmacêutica. São Paulo, v.42, n.3, p.455460, jul./set. 2006.

TAKAYANAGUI, O. M. et al. Fiscalização de verduras comercializadas no município de Ribeirão Preto, SP. Revista da Sociedade Brasileira de Medicina Tropical. v.34, n.1, p.37-41, jan-fev, 2001

TRAVIEZO-VALLES et al. Contaminación enteroparasitaria de lechugas expendidas en mercados del estado Lara. Parasitologia Latinoamericana, Santiago, v. 59, p.167-70, 2004.

VALLADA, E. P ; SEIXAS, A. S.S; CARRARO, K.M.A. Contribuição ao estudo da transmissão das enteroparasitas. Revista Brasileira de Farmácia, v.68, p. 102-108, 1987.

VOLLKOPF, P.C.P.; LOPES, F.M.R.; NAVARRO, I.T. Enteroparasites occurrence in lettuce samples (Lactuca sativa) commercialized in Porto Murtinho - MS. Arq. ciên. vet. zool. UNIPAR, Umuarama, v. 9, n. 1, p.37-40, 2006. 